\title{
Time Variability Studies with ROSAT
}

\author{
Martin Kürster \\ Max-Planck-Institut für extraterrestrische Physik, D-85740 Garching, Germany
}

\begin{abstract}
An overview over the mission of the X-ray astronomy satellite ROSAT (Röntgensatellit) is given with an emphasis on the first soft X-ray all-sky survey carried out with an imaging telescope. Several examples of time variability studies made possible by the all-sky survey are shown for active late-type stars. Two exceptional long-duration stellar flares are presented.
\end{abstract}

\section{ROSAT mission overview}

At present the X-ray astronomy satellite ROSAT is in the fifth year of its very successful mission. ROSAT is an observatory in a low-earth orbit carrying an $\mathrm{X}$ ray telescope (XRT; Trümper 1983, Aschenbach 1988) and an XUV telescope, the 'Wide Field Camera' (WFC; Wells et al. 1990, Kent et al. 1990). Both telescopes are assemblies of nested Wolter type I mirrors.

The focal plane instrumentation of the XRT comprises two identical Position Sensitive Proportional Counters (PSPC) built by the Max-Planck-Institut für extraterrestrische Physik (MPE; Pfeffermann et al. 1988), and the High Resolution Imager (HRI), a microchannel plate detector built by the Smithsonian Astrophysical Observatory (SAO; David et al. 1993). One of the PSPCs was lost early in the mission, however, due to the redundant second PSPC this did not constitute any impairment of the project. Recently, the supply of PSPC detector gas was used up, so that the PSPC guest observer program had to be ended in July 1994. Currently, the XRT observations are continued with the HRI.

Properties of the PSPC are a large field of view of $2^{\circ}$ diameter, a very low intrinsic background of $2 \times 10^{-5} \mathrm{cts} \mathrm{sec}^{-1} \operatorname{arcmin}^{-2}$, and a moderate spectral resolution of about 6 independent resolution elements over its sensitive energy range $0.1-2.4 \mathrm{keV}$. At $1 \mathrm{keV}$ the PSPC yields an effective area of $220 \mathrm{~cm}^{2}$. Contrary to the PSPC, the HRI is lacking any spectral resolution, but its spatial resolution of $\approx 3-4^{\prime \prime}$ surpasses that of the PSPC $\left(\approx 20^{\prime \prime}\right.$ on-axis $)$ by a factor of $5-7$. The field of view of the HRI has a diameter of $40^{\prime}$.

After ROSAT's launch on June 1, 1990, and a 2-month calibration-verification phase half a year of the mission was devoted to the first all-sky survey to be carried out with an imaging telescope in soft X-rays (see Sect. 2). Simultaneous to the XRT survey the WFC carried out the first all-sky survey in the XUV 


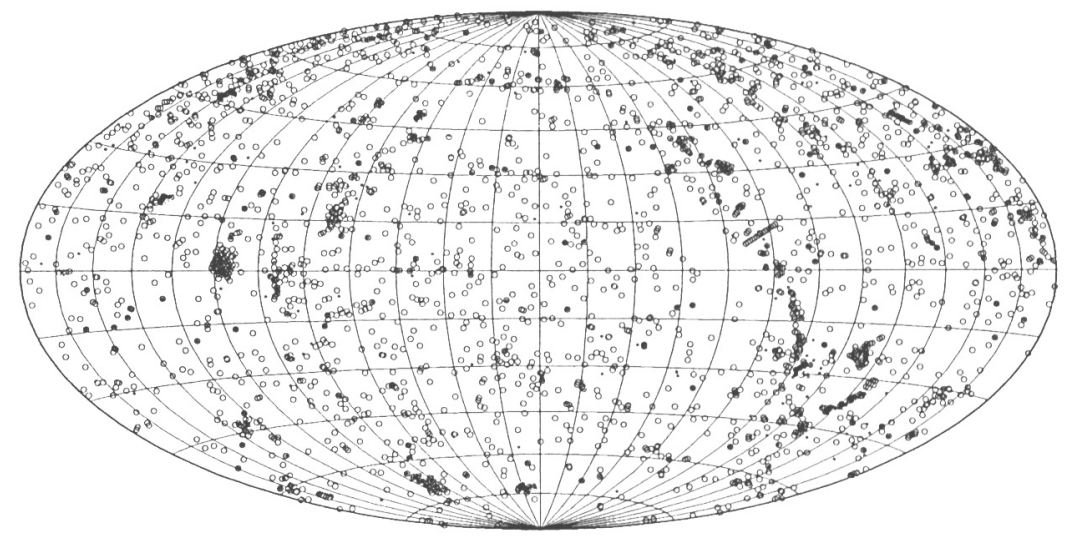

Fig. 1. Distribution of 3987 sky fields observed by ROSAT in pointed mode up to Dec 3, 1993. An Aitoff equal-area whole-sky projection is shown in equatorial coordinates; with respect to the center at $\mathrm{RA}=0, \mathrm{DEC}=0$, north is up and east is to the left.

$(0.025-0.2 \mathrm{keV})$. However, no WFC observations will be discussed here. An overview over the XUV survey can be found in Page \& Denby (1992); the WFC catalogue of bright survey sources was published by Pounds et al. (1993). Since February 1991 ROSAT has made pointed observations of over 5000 fields. Fig. 1 shows their celestial distribution; it is complete up to December 3, 1993.

About 60,000 sources were detected in the XRT all-sky survey (see Sect. 2), and another 60000 sources were discovered by pointed observations. Proposals for pointed ROSAT observations were accepted from about 540 PIs from 24 countries. After one year of PI proprietary time all pointed data enter public archives at MPE, GSFC, and in Leicester. Currently about 600 archival requests per month demonstrate the high interest of the scientific community in ROSAT data. More than 500 publications in refereed journals have reported ROSAT results.

With respect to the topic of this conference it should be pointed out that ROSAT was not primarily devised for time variability studies. There are two complications for any kind of time dependent observation. First, the fixed mount of the solar panels constrains telescope orientation to a $30^{\circ}$ wide strip on the sky perpendicular to the solar direction; and second, the low earth orbit causes frequent earth blocks of targets typically once per orbit. For this reason only $5 \%$ of the total pointed observing time is reserved for time critical observations.

Fortunately, observations that are more or less randomly distributed in time have proved to allow very successful studies of time variable phenomena; one observation of this type will be shown in Sect.3. Additionally, the scanning approach adopted in the all-sky survey provided another unique opportunity for time variability studies; three examples taken from the survey will be given in Sects. 2 and 3. All examples concern coronally active late-type stars. 


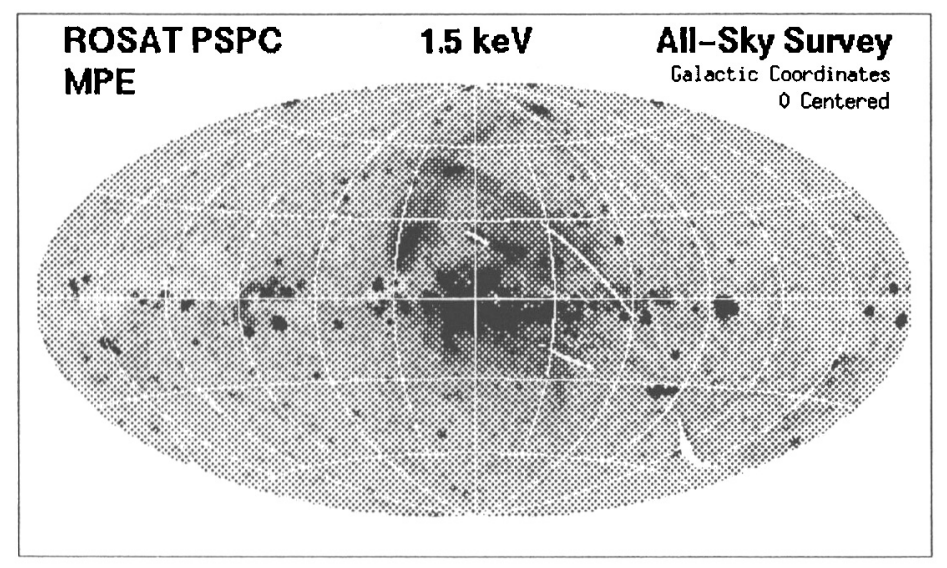

Fig. 2. The ROSAT X-ray sky in the hard energy band $(1.0-2.0 \mathrm{keV})$. This image from the all-sky survey is displayed in galactic coordinates centered on latitude $0^{\circ}$ and longitude $0^{\circ}$. The hard band was selected because of its low background.

\section{The ROSAT XRT all-sky survey}

For the ROSAT X-ray all-sky survey the sky was scanned in great circles (one circle per $96 \mathrm{~min}$ satellite orbit) whose normal pointed at the Sun. Following the Sun over half a year the whole sky was covered. Each scan collected photons from a sky strip of width $2^{\circ}$ equal to the size of the PSPC's field of view.

Due to this scanning geometry the poles of the ecliptic were contained in every scan and observed in $96 \mathrm{~min}$ steps over half a year whereas sky regions near the ecliptic equator were covered only during a 2 day interval. Exposure times for the ecliptic poles were $\approx 50000 \mathrm{sec} v \mathrm{vs} \approx 400 \mathrm{sec}$ for the ecliptic equator. Satellite passages through the Earth's radiation belts whose high particle background requires to switch off the detector, decreased the exposure locally making the overall distribution inhomogeneous. Due to the South Atlantic Anomaly (SAA) southern hemisphere exposure times were shorter than northern hemisphere ones. More details on the survey are given by Voges $(1992,1993)$.

Fig. 2 shows the exposure corrected all-sky image obtained from the survey in the hard energy band $(1.0-2.0 \mathrm{keV})$ displayed in galactic coordinates (Snowden et al. 1995). The bright point sources (mostly accreting binaries) can be seen to accumulate in the galactic plane and in the Large Magellanic Cloud (lower right). Other prominent sources are supernova remnants and clusters of galaxies, e.g. the Virgo cluster at high galactic latitudes (top right). Galactic diffuse emission is most pronounced near the center region, in the Cygnus Loop (ringlike feature at center left) and in the North Polar Spur (arclike region in upper half of image).

The celestial distribution of survey point sources as detected by the ROSAT Standard Analysis System (SASS) in the total energy band is shown in Fig. 3. 


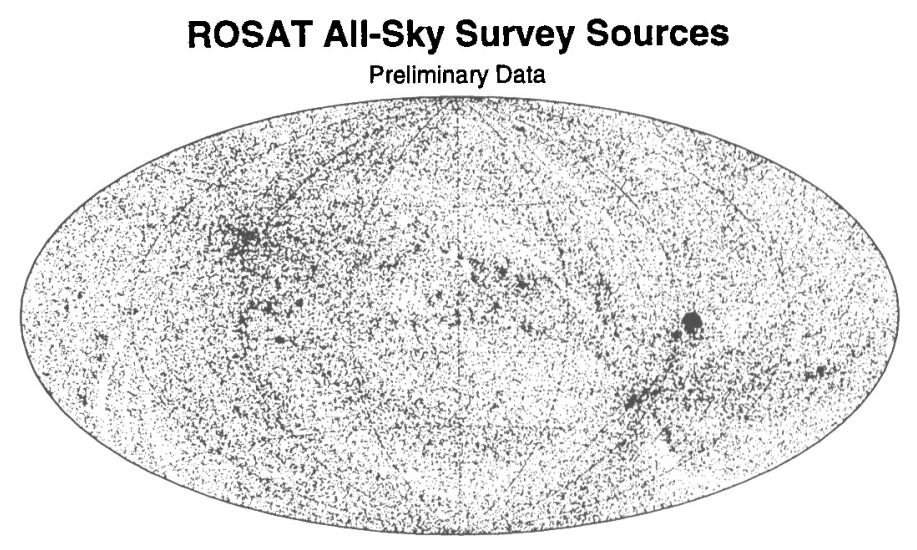

Fig. 3. Point sources detected in the ROSAT X-ray all-sky survey in the total energy band $(0.1-2.4 \mathrm{keV}$ ) and displayed in galactic coordinates (as Fig. 2). The exposure time varies strongly over the sky with a mean value of $400 \mathrm{sec}$ corresponding to a flux limit of $5 \times 10^{-13} \mathrm{erg} \mathrm{cm} \mathrm{cm}^{-2} \mathrm{sec}^{-1}$. This preliminary image containing 58359 sources will be improved after a reprocessing of the survey data.

Enhanced sensitivity at the ecliptic poles shows as particularly dense source regions near the centers of the 2nd and 4th quadrants (upper left and lower right).

Figs. 4 and 5 demonstrate the typical temporal coverage of the sky showing survey light curves of two X-ray sources which are located near the ecliptic equator and near the southern ecliptic pole, respectively. Both objects are rapidly rotating, coronally active stars and, among these, prime candidates for time variability to occur in the form of flares and of rotational modulation caused by an inhomogeneous distribution of coronal emission regions.

Fig. 4 shows the light curve of HR 1099 (=V711 Tau), an evolved RS CVntype binary (spectral types G5IV+K1IV) in a 2.8 day synchronized orbit. Data points correspond to single survey scans in an observing window 2 days wide. A short-duration flare is seen in a single scan in an otherwise constant light curve. This low level of variability in a very active binary came as a surprise.

Fig. 5 shows the light curve of the single pre-main sequence $\mathrm{KOV}$ dwarf AB Dor (=HD 36705) which was observed over 35 days, however, with frequent data gaps mostly due to the SAA. The source is very variable exhibiting flaring activity as well as variability on time scales of a few hours. A period analysis failed to find the $\mathbf{1 3}$ hour rotation period implying that most of the variability is 'irregular,' i.e. attributable to low-level flaring and/or restructuring of coronal emission regions on time scales shorter than the rotation period (see Kürster et al. 1992). Pagano et al. (1993) compare this data set with simultaneous observations made in other wavelength bands. Rotational modulation in AB Dor is revealed in a pointed observation presented by Kürster \& Dennerl (1993).

More on survey observations of active late-type stars can be found in papers by Dempsey et al. (1993a,b), Schmitt (1994), and Haisch \& Schmitt (1994). 


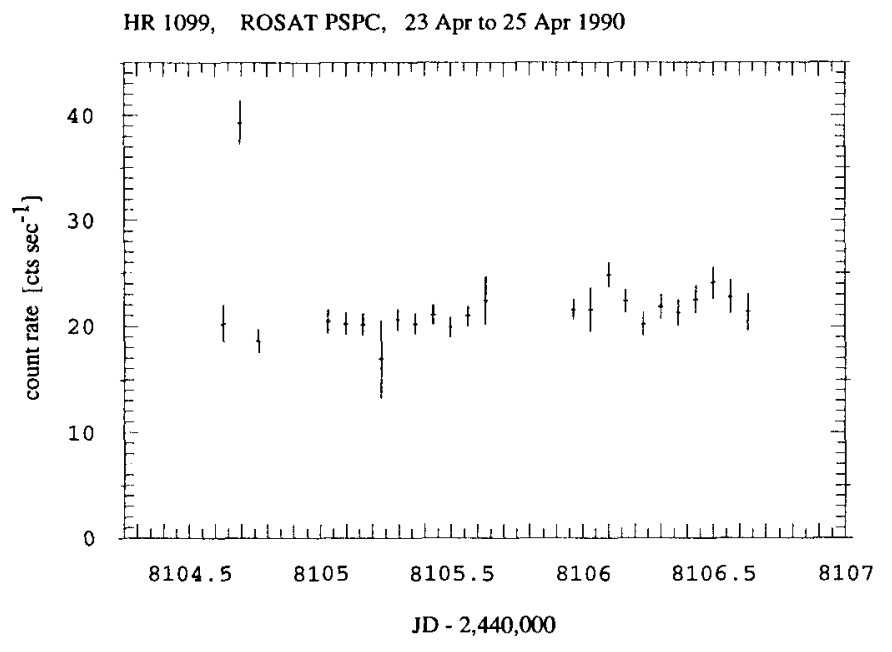

Fig. 4. ROSAT all-sky survey light curve of the RS CVn-type binary HR 1099 (=V711 Tau). Data points are spaced at 96 min intervals and represent individual scans. Being located at a low ecliptic latitude $\left(-18.3^{\circ}\right)$ HR 1099 was visible only for 2 days. A short-duration flare occured in an otherwise non-variable episode of the star.

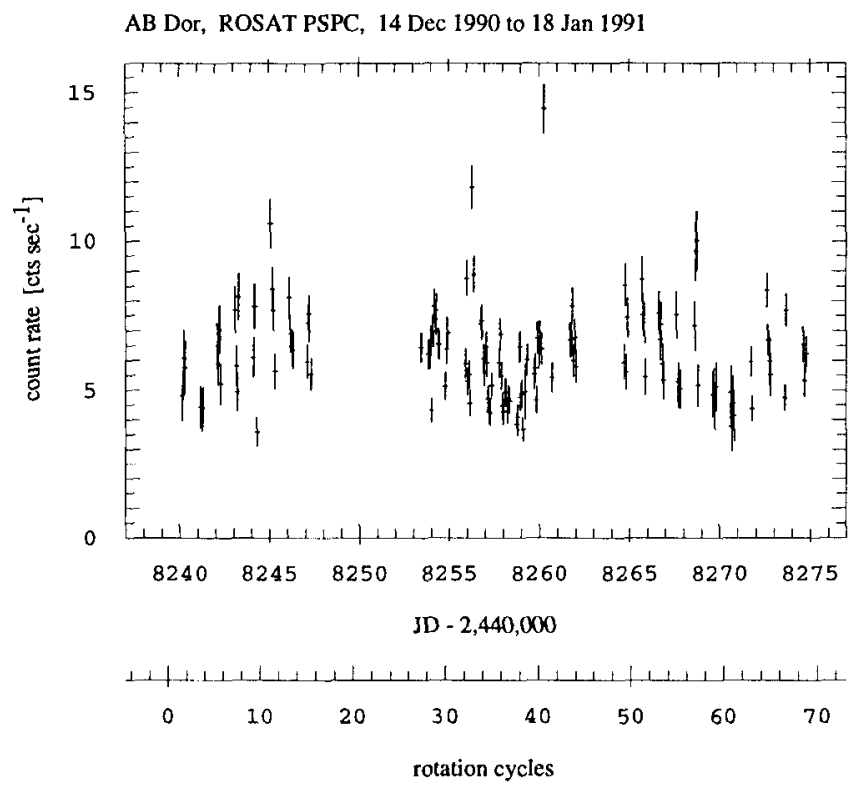

Fig. 5. ROSAT all-sky survey light curve of the young KOV star AB Dor (=HD 36705) which is located within $3.4^{\circ}$ of the south ecliptic pole and was visible during the survey for about 35 days or almost 70 rotational cycles $(P=0.51479$ day; Innis et al. 1988). Most of the many data gaps are due to detector switch-offs in the SAA. 
HD 197890, ROSAT PSPC, 9/10 July 1990

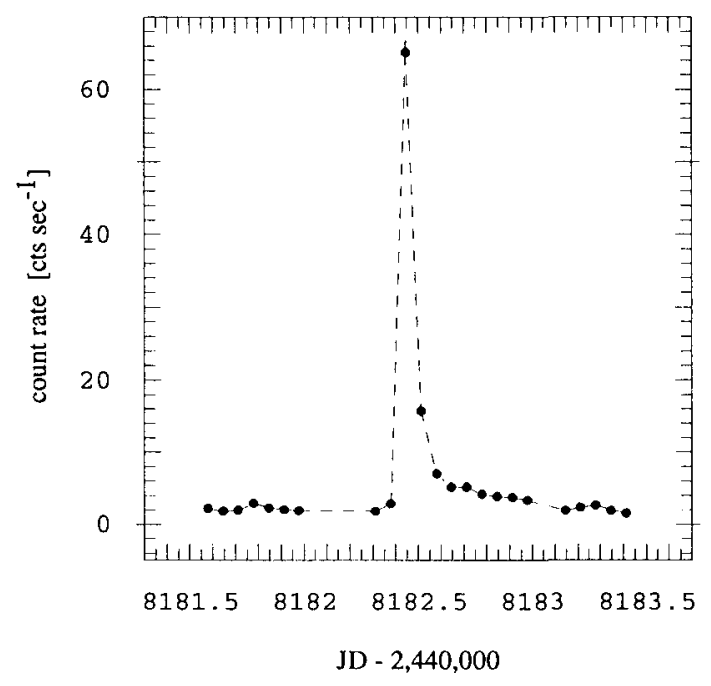

Fig. 6. ROSAT all-sky survey observation of the AB Dor-type KoV star HD 197890 (='Speedy Mic') showing the brightest stellar X-ray flare seen in the survey. The total flare duration is about twice the stellar rotation period.

\section{Two exceptional long-duration stellar flares}

This section presents two highlights among time variability studies carried out with ROSAT. Both are long-duration flares on active stars. To begin with the conclusion, in both cases the flares lasted several stellar rotations with no strong modulation of the flare light curve visible; deep depressions in the light curve are expected if the rotating star takes the flaring region to its invisible hemisphere. In both events the flaring regions must either have been very extended vertically, so that they were not totally occulted, or located in the small circumpolar regions of these high inclination stars. Both scenarios are quite different from solar flares which occur in rather compact regions and never near the poles.

The first observation revealed the strongest (in terms of the peak count rate) stellar X-ray flare found in the all-sky survey. It occured on the young AB Dor-type KOV star HD 197890 in Microscopium, nicknamed 'Speedy Mic' due to its very high rotational velocity. This event was first discovered by Matthews et al. (1994) in the WFC all-sky survey in which the source was detectable only during the flare phase. The XRT survey data taken over a 2 day interval revealed flaring emission as well as quiescent emission before and after the outburst (Fig. 6; see also Kürster 1994a). The flare released an energy of $\approx 2.2 \times 10^{36} \mathrm{erg} \mathrm{sec}^{-1}$ in the PSPC band. Its duration of 0.7 day is about twice the stellar rotation period of 0.3 day (Anders et al. 1993). No occultationtype modulation is seen during the flare implying that it either occured in a 


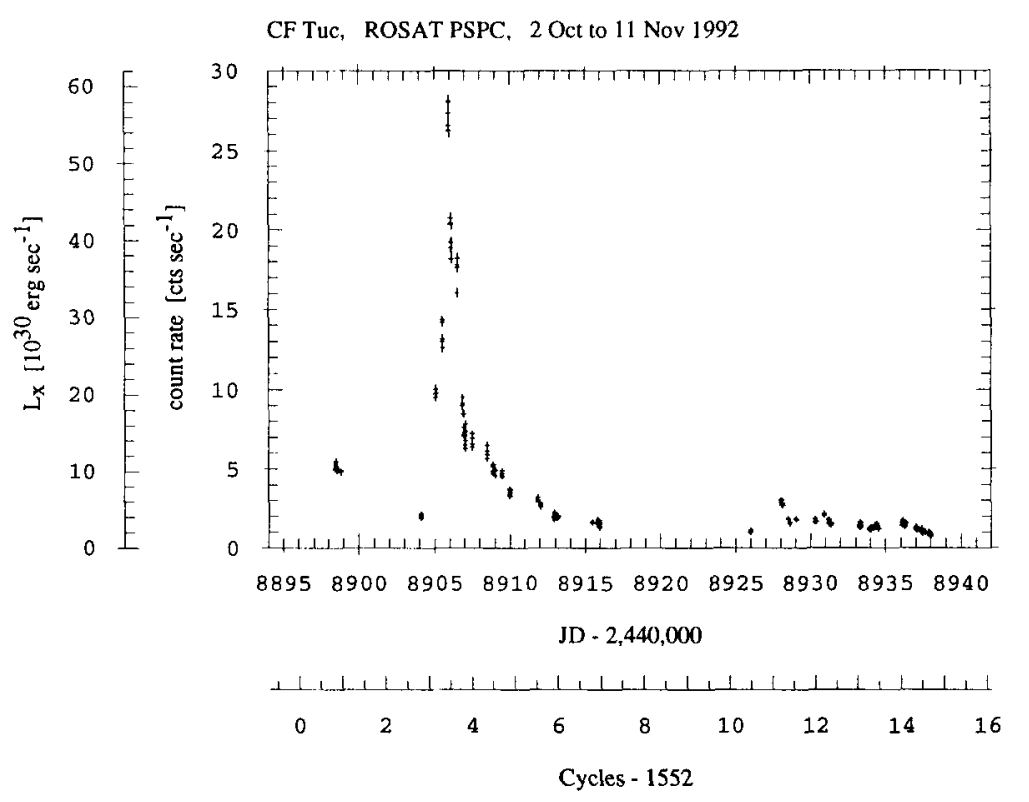

Fig. 7. A pointed observation of the RS CVn-type binary CF Tuc (=HD 5303) showing the longest known stellar X-ray flare which lasted more than 3 rotational/orbital binary cycles (ephemeris $H J D 2,444,555.009+2.797672$ day; period from Budding 1985; time of primary eclipse refined). The X-ray luminosity assuming a constant flux conversion factor of $6.0 \times 10^{-12} \mathrm{erg} \mathrm{cm}^{-2} \mathrm{cts}^{-1}$ is indicated.

very extended region or on the pole. However, the quiescent emission (before JD $2,448,182$ and after JD 2,448,183) shows a slight modulation compatible with the 0.3 day period indicating an inhomogeneous corona.

In a pointed observation extending over 40 days the second flare (Fig. 7) was discovered on the partially eclipsing RS CVn-type binary CF Tuc (=HD 5303; spectral types G0IV+K4IV). It is the longest $\mathrm{X}$-ray flare ever observed with a total duration of 9 days or more than three times the 2.8 day rotational/orbital period of this synchronized binary. With an energy release of $\approx 7.8 \times 10^{36} \mathrm{erg} \mathrm{sec}^{-1}$ in the PSPC band this outburst is also among the strongest known X-ray flares. As for 'Speedy Mic', there are no indications for rotational occultations of the flaring region nor for eclipses by the companion star (see Kürster 1994a,b).

These temporally extended observations made by ROSAT in the all-sky survey or in widespread pointed observations provide unique opportunities for detailed studies of long-duration stellar flares. I have shown two examples that yield important information on the structure of active-star coronae from their mere duration. Other stellar flares observed with ROSAT in pointed mode can be found in papers by Ottmann \& Schmitt (1994), and Preibisch et al. (1993). 
Acknowledgements: The ROSAT project has been a collaborative effort of many people at MPE, AIT, GSFC, SAO, and the UK WFC consortium. The work described in this paper was most directly supported by J. Trümper, W. Voges, and J. Schmitt. K. Dennerl and S. Snowden provided some figures. Special thanks to the MPE ROSAT data center, in particular R. Gruber, W. Pietsch, C. Rosso, F. Haberl, and U. Zimmermann. The ROSAT project is supported by the German Bundesministerium für Forschung und Technologie (BMFT/DARA) and the Max Planck Society.

\section{References}

Anders G.J., Jeffries R.D., Kellett B.J., Coates D.W., 1993, MNRAS 265, 941

Aschenbach B., 1988, Appl. Optics 27, 1404

Budding E., 1985, IBVS 2779

David L.P., Harnden F.R., Kearns K.E., Zombeck M.V., 1993, The ROSAT High Resolution Imager (HRI), U.S. ROSAT Science Data Center/SAO document

Dempsey R.C., Linsky J.L., Fleming T.A., Schmitt J.H.M.M., 1993a, ApJSS 86, 599

Dempsey R.C., Linsky J.L., Schmitt J.H.M.M., Fleming T.A., 1993b, ApJ 413, 333

Haisch B., Schmitt J.H.M.M., 1994, ApJ 426, 716

Innis J.L., Thompson K., Coates D.W., Lloyd Evans T., 1988b, MNRAS, 235, 1411

Kent B.J., Reading D.H., Swinyard, B.M., Graper E.B., Spurett P.H., 1990, Proc. SPIE, Vol. 1344, p. 255

Kürster M., 1994a, AG Abstract Series 10, 22

Kürster M., 1994b, in Proc. 8th Cambridge Workshop on Cool Stars, Stellar Systems, and the Sun, J. Linsky (ed.), A.S.P. Conf. Ser. 64, p. 104

Kürster M., Dennerl K., 1993, in Physics of Solar and Stellar Coronae, ed. J. Linsky and S. Serio (Kluwer, Dordrecht), p. 443

Kürster M., Schmitt J.H.M.M., Fleming T.A., 1992, in Proc. 7th Cambridge Workshop on Cool Stars, Stellar systems and the Sun, M. Giampapa and J. Bookbinder (eds.), A.S.P. Conf. Ser. 26, p. 109

Matthews L., et al., 1994, MNRAS 266, 757

Ottmann R., Schmitt J.H.M.M., 1994, A\&A 283, 871

Pagano I., Rodonó M., Cutispoto G., Collier Cameron A., Kürster M., Kellet B.J., Bromage G.E., Jeffries R., Foing B., Ehrenfreund P., 1993, in Physics of Solar and Stellar Coronae, J. Linsky \& S. Serio (eds.), Kluwer, Dordrecht, p. 457

Page C.G. Denby M., 1992, in Proc. 1st Ann. Conf. on Astonomical Data Analysis Software and Systems, (in press)

Pfeffermann, E., Briel U.G., Hippmann H., Kettenring G., Metzner G., Predehl P., Reger G., Stephan K.H., Zombeck M.V., Chappell J., \& Murray S.S., 1988, Proc. SPIE 733, 519

Pounds K.A, et al., 1993, MNRAS 260, 77

Preibisch Th., Zinnecker H., Schmitt J.H.M.M., 1993, A\&A 279, L33

Schmitt J.H.M.M., 1994, ApJS 90, 735

Snowden S.L., Freyberg M.J., Schmitt J.H.M.M., Voges W., Trümper J., Edgar R.J., McCammon D., Plucinsky P.P., Sanders W.T., 1995, ApJ (in press)

Trümper J., 1983, Adv. Space Res. Vol. 2, No. 4, p. 241

Voges W., 1992, in Proc. ISY Conf. "Space Science", ISY-3, ESA publications, p. 9

Voges W., 1993, Adv. Space Res., Vol 13, No. 12, p. 391

Wells A. et al., 1990, Proc. SPIE, 1344, p. 230 